\title{
Effects of Tailored Surface Chemistry on Desorption Electrospray lonization Mass Spectrometry: a Surface-Analytical Study by XPS and AFM
}

\author{
Andrea Penna, ${ }^{1,3}$ Maria Careri, ${ }^{1}$ Nicholas D. Spencer, ${ }^{3}$ Antonella Rossi ${ }^{2,3}$ \\ ${ }^{1}$ Dipartimento di Chimica, Università di Parma, Parco Area delle Scienze 17/A, 43124, Parma, Italy \\ ${ }^{2}$ Dipartimento di Scienze Chimiche e Geologiche, Università di Cagliari, Complesso Universitario di Monserrato, Unità di Ricerca \\ INSTM, 09042, Monserrato, Cagliari, Italy \\ ${ }^{3}$ Department of Materials, ETH Zurich, Vladimir-Prelog-Weg 5, 8093, Zürich, Switzerland
}

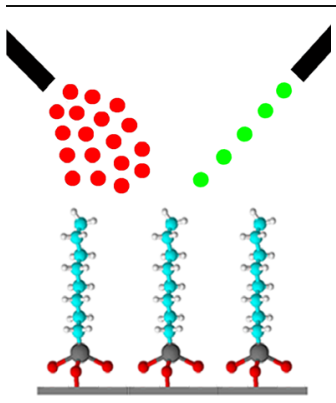

Abstract. Since it was proposed for the first time, desorption electrospray ionizationmass spectrometry (DESI-MS) has been evaluated for applicability in numerous areas. Elucidations of the ionization mechanisms and the subsequent formation of isolated gas-phase ions have been proposed so far. In this context, the role of both surface and pneumatic effects on ion-formation yield has recently been investigated. Nevertheless, the effect of the surface chemistry has not yet been completely understood. Functionalized glass surfaces have been prepared, in order to tailor surface performance for ion formation. Three substrates were functionalized by depositing three different silanes [3-mercaptopropyltriethoxysilane (MTES), octyltriethoxysilane (OTES), and $1 \mathrm{H}, 1 \mathrm{H}, 2 \mathrm{H}, 2 \mathrm{H}$-perfluorooctyltriethoxy-silane (FOTES)] from toluene solution onto standard glass slides. Surface characterization was carried out by contact-angle measurements, tapping-mode atomic force microscopy, and X-ray photoelectron spectroscopy. Morphologically homogeneous and thickness-controlled films in the $\mathrm{nm}$ range were obtained, with surface free energies lying between 15 and $70 \mathrm{~mJ} / \mathrm{m}^{2}$. These results are discussed, together with those of DESI-MS on lowmolecular-weight compounds such as melamine, tetracycline, and lincomycin, also taking into account the effects of the sprayer potential and its correlation with surface wettability. The results demonstrate that ionformation efficiency is affected by surface wettability, and this was demonstrated operating above and below the onset of the electrospray.

Keywords: Desorption electrospray ionization, Mass spectrometry, Ionization mechanism, Surface functionalization, Self-assembled monolayers, X-ray photoelectron spectroscopy

Received: 31 December 2014/Revised: 6 March 2015/Accepted: 6 March 2015/Published Online: 21 April 2015

\section{Introduction}

A large number of papers have highlighted desorption electrospray ionization-mass spectrometry (DESI-MS) as a high-throughput technique with a variety of applications $[1,2]$, recently assessed as reliable technique for quantitative analysis [3]. Several papers have been published on the ionization mechanisms leading to the formation of isolated gas-

Electronic supplementary material The online version of this article (doi:10.1007/s13361-015-1135-9) contains supplementary material, which is available to authorized users.

Correspondence to: Andrea Penna; e-mail: andrea.penna@gmx.com, Antonella Rossi; e-mail: rossi@unica.it phase ions, supporting the hypothesis of a two-step, dropletpick-up model followed by ESI-like desolvation and gas-phase ion formation by the action of the electric field [4-7]. In this context, the roles of surface and pneumatic effects on ionformation yield have recently been examined, by investigating surface charge $[8,9]$, hydrodynamic and pneumatic forces [1013], and interactions between analytes and the surface [14]. In the present study, surface-chemistry effects on DESI-MS response have been thoroughly investigated and correlated with the ion-formation yield. It is known that the electrical properties of the substrate strongly influence the mass spectrometric response, in terms of magnitude of the steady-state current and different capacitor time constants of the equivalent $\mathrm{RC}$ circuits [8]. Therefore, the electrical contribution of the substrate (i.e., electrical resistivity), was set to be constant, by using the same 
material as a support for the functionalization reactions, in order to address solely the surface-chemical contributions to ion formation.

One of the most successful approaches for the modification of solid substrates has been the chemical grafting of hydrocarbon chains on hydrated surfaces via an alkylchlorosilane $\left(-\mathrm{SiCl}_{3}\right)$ or alkylalkoxysilane (-Si $\left.\{\mathrm{OR}\}_{3}\right)$ head group by the formation of self-assembled monolayer structures [15-18]. However, despite extensive efforts, the silanization reaction could still be affected by irreproducibility in the macroscopic properties (i.e., wettability and contact angle hysteresis) of the deposited film, when experimental variables such as the nature of the substrate [19], temperature [20], concentration [21, 22], solvent type [23], and water content [24, 25] are not under strict control. By controlling these parameters, it can be possible to obtain a structure of alkylsilane monolayers with good reproducibility because agglomeration during the deposition is minimized [26, 27].

Taking into account the aforementioned knowledge about surface functionalization and starting from our previously published results [14], the aim of this paper is to address further questions regarding surface effects in the DESI mechanism. For this purpose, we set up an experimental strategy based on the synthesis and the characterization of differently functionalized glasses, by means of three different silanes (Figure 1), [i.e., mercaptopropyl-triethoxysilane (MTES), octyl-triethoxysilane (OTES), and $1 \mathrm{H}, 1 \mathrm{H}, 2 \mathrm{H}, 2 \mathrm{H}$-perfluorooctyl-triethoxysilane (FOTES)], with glass itself used as a hydrophilic surface, in order to obtain surfaces covering a wide range of surface free energy. Surface characterization was carried out by means of tapping-mode atomic force microscopy (TM-AFM), contactangle measurements, and X-ray photoelectron spectroscopy (XPS).

Finally, these four supports were used for pneumatically assisted DESI-MS experiments for the analysis of mixtures of small molecules with widely known acid-base behavior and deposited on all substrates. In particular, melamine (organic base, MW 126), lincomycin (lincosamide antibiotic, MW 406)

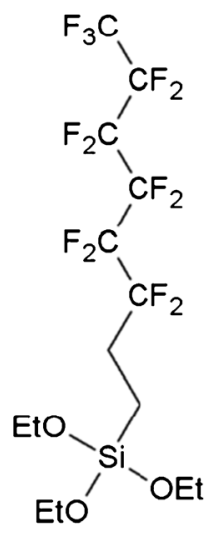

(a)

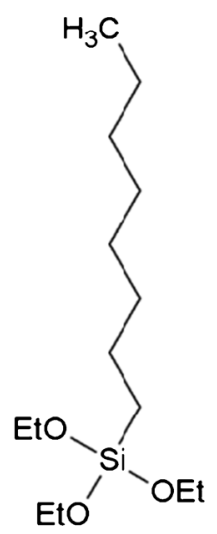

(b)

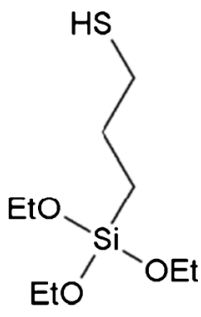

(c)
Figure 1. Chemical formulae of silanes adsorbed onto glass slides, i.e., (a) FOTES, (b) OTES, and (c) MTES and tetracycline (polyketide antibiotic, MW 444) (Figure 2) were considered. The use of functionalized surfaces allowed the role of surface chemistry to be investigated under different MS conditions.

\section{Experimental}

\section{Materials}

Glass microscope slides $(76 \mathrm{~mm} \times 25 \mathrm{~mm} \times 1 \mathrm{~mm})$ were provided by Thermo Scientific (Wohlen, Switzerland). 3Mercaptopropyl-triethoxysilane (MTES) was obtained from Alfa Aesar (Ward Hill, MA, USA), octyltriethoxysilane (OTES) from Acros (Acros Organics, Geel, Belgium), and 1H,1H,2H,2H, perfluorooctyl-triethoxysilane (FOTES) from ABCR-Chemicals (Karlsruhe, Germany). Methanol, acetonitrile, and toluene were from Sigma-Aldrich (St. Louis, MO, USA). Ethanol was from Scharlau (analytical grade, Barcelona, Spain). Deionized (DI) water was purified to $18.2 \mathrm{M} \Omega \cdot \mathrm{cm}$ using a Milli-Q water purification system (Milli-Q element A10 System; Millipore, San Francisco, CA, USA). Melamine, lincomycin, and tetracycline were obtained from SigmaAldrich (St. Louis, MO, USA).

\section{Silanization}

Glass microscope slides were used as supports for each film synthesis. All materials were cleaned by ultrasonication, first for $10 \mathrm{~min}$ in toluene, then twice for $10 \mathrm{~min}$ in ethanol, and then they were blown dry in a stream of nitrogen. Subsequently, the supports were cleaned for $2 \mathrm{~min}$ in oxygen plasma (Harrick Plasma, Ithaka, NY, USA) at a pressure of 0.03 mbar. All microscope slides were then dipped for $1 \mathrm{~h}$ into silane solutions in dry toluene: $100 \mathrm{mM}$ solutions for MTES and OTES and $5 \mathrm{mM}$ for FOTES. Each silanized surface was rinsed with water, then with toluene. Samples were annealed at $100^{\circ} \mathrm{C}$ under reduced pressure of $50 \mathrm{mbar}$, for $60 \mathrm{~min}$.

\section{Surface Characterization}

Contact-Angle Goniometry and Surface-Free-Energy Calculations The equilibrium contact angle of a sessile drop $(5 \mu \mathrm{L})$ was measured with a contact-angle goniometer (RaméHart model 100; Ramé-Hart, Inc., Mountain Lakes, NJ, usa) for

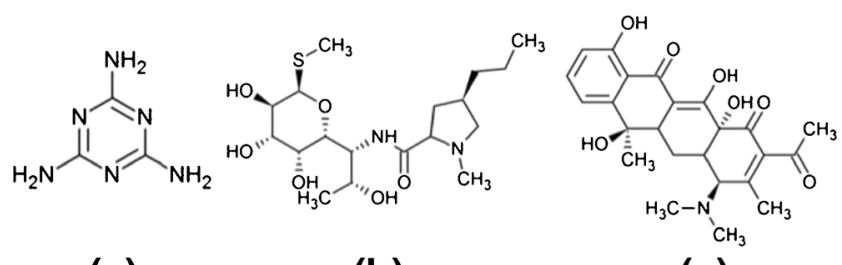

(a)

(b)

(c)

Figure 2. Chemical formulae of the compounds used for DESI-MS 
each surface treatment. The average contact angle was determined by the measurement of 10 drops. The Owens-WendtKaelble (OWK) [28] method was used to calculate surface energies.

In the OWK method, the polar $\left(\gamma_{S}{ }^{p}\right)$ and dispersion $\left(\gamma_{S}{ }^{d}\right)$ components of the surface energies of functionalized slides were determined by Equations 1 and 2 .

$\gamma_{L}(1+\cos \theta)=2\left(\sqrt{\gamma_{S}^{d} \gamma_{L}^{d}}+\sqrt{\gamma_{S}^{p} \gamma_{L}^{p}}\right)$

$\gamma_{S}=\gamma_{S}^{p}+\gamma_{S}^{d}$

The parameters $\gamma_{L}, \gamma_{L}{ }^{d}$, and $\gamma_{L}^{p}$ refer to the total surface tension, and the dispersion and polar components of the probe liquid, respectively. The surface energy $\left(\gamma_{S}\right)$ of a modified surface is the sum of polar $\left(\gamma_{S}^{p}\right)$ and dispersion $\left(\gamma_{S}{ }^{d}\right)$ components of the solid.

The contact angles were measured using two liquids, that is, water and diiodomethane (DM). The corresponding surface tensions $\left(\gamma_{L}\right)$ are 72.8 and $50.8 \mathrm{mN} / \mathrm{m}$, respectively. Polar and dispersion components of the surface tension were from $\mathrm{Yu}$ and Dekker [29].

Tapping-Mode Atomic Force Microscopy (TM$A F M)$ Morphologies of hybrid-silica films were investigated by a DI Dimension atomic force microscopy (Digital Instrument, Santa Barbara, CA, USA) in tapping mode at room temperature with a silicon nitride cantilever (Olympus, Shinjuku, Tokyo, Japan), which had a resonant frequency of $265 \mathrm{kHz}$ and a spring constant of $25.5 \mathrm{~N} / \mathrm{m} ; 10 \mu \mathrm{m} \times 10 \mu \mathrm{m}$ images were acquired, rms roughness $\left(\mathrm{R}_{\mathrm{q}}\right)$ calculated for each surface, and then averaged over three independent investigations.

X-ray Photoelectron Spectroscopy The surface chemistry of silica films on glass supports was investigated by means of X-ray photoelectron spectroscopy (XPS), using a Theta Probe (Thermo Fisher Scientific, East Grinstead, UK). A monochromatic $\mathrm{Al} \mathrm{K} \alpha$ source with a beam diameter varying between 15 and $400 \mu \mathrm{m}$ was used. The electrons emitted from the sample surface were collected with a radian lens having an acceptance angle of $60^{\circ}$ (ranging from $23^{\circ}$ to $83^{\circ}$ emission angle) and, after passing the hemispherical analyzer, were detected by a two-dimensional detector. The average emission angle was $53^{\circ}$. The system was also equipped with an argon-ion gun and a combined low-energy electron/ ion flood gun for charge compensation. Angle-resolved XPS (ARXPS) analyses were carried out at 16 emission angles using the monochromatic $\mathrm{Al} \mathrm{K} \alpha$ source with a beam diameter of $300 \mu \mathrm{m}$ and a power of $70 \mathrm{~W}$ in the constantanalyzer-energy (CAE) mode. The pass energy and the step size were, respectively, 100 and $0.1 \mathrm{eV}$ [full width at halfmaximum (FWHM) of the peak height for $\mathrm{Ag} 3 \mathrm{~d}_{5 / 2}=$ $0.84 \mathrm{eV}]$. Survey spectra were acquired with pass energy of $300 \mathrm{eV}$ and a step size of $1 \mathrm{eV}$. A low-energy electron/ ion flood gun for charge compensation was used with electron energy $-1.6 \mathrm{~V}$ at $7 \cdot 10^{-7} \mathrm{mbar}$. The spectrometer was calibrated according to ISO 15472:2001 with an accuracy of $\pm 0.1 \mathrm{eV}$. The high-resolution spectra were processed using CasaXPS software (Casa Software Ltd., Wilmslow, Cheshire, UK). Minor charging was corrected by referencing to aliphatic carbon at $285.0 \mathrm{eV}$. Peak fitting was performed after a linear type background subtraction using $30 \%$ Lorentzian $70 \%$ Gaussian model functions. Data processing was carried out constraining the $2 p_{3 / 2}$ and $2 p_{1 / 2}$ energy difference of $\mathrm{Si} 2 \mathrm{p}$ and $\mathrm{S} 2 \mathrm{p}$ equal to $0.7 \mathrm{eV}$ and $1.3 \mathrm{eV}$ respectively.

Quantitative analysis was performed on the basis of the integrated intensity, obtained from the original spectra after background subtraction and curve synthesis, using a firstprinciple approach and applying the equations of Powell [30]. The apparent atomic concentration was calculated as:

$X_{j}=\frac{\frac{I_{i j}}{S_{i j}}}{\sum \frac{I_{i j}}{S_{i j}}}$

where $I_{i j}$ and $S_{i j}$ are the area and the sensitivity factor of the peak $i$ of the element $j$, respectively. The sensitivity factors were calculated from Scofield's photoionization cross-section [31], the angular asymmetry factor [32], the spectrometer transmission function, and the inelastic mean free path (IMFP) corrected for the emission angle, assuming the sample to be homogeneous. The inelastic mean free path was calculated using the equation proposed by Seah and Dench [33].

Desorption Electrospray-Mass Spectrometry (DESI-MS) A linear ion trap LTQ Orbitrap XL mass spectrometer (Thermo Fisher Scientific, San Josè, CA, USA) equipped with a DESI ion source (Prosolia Inc., Indianapolis, IN, USA) was used. The source parameters were set as follows: solvent flow-rate, $2 \mu \mathrm{L} \mathrm{\text {min}^{-1 }}$; nitrogen pressure, 150 psi; capillary voltage, $20 \mathrm{~V}$; tube lens voltage, $40 \mathrm{~V}$; injection time, $100 \mathrm{~ms}$; tip-to-surface distance, $2 \mathrm{~mm}$; tip-to-inlet distance, $4 \mathrm{~mm}$; incidence angle, $54^{\circ}$. The capillary temperature was $250^{\circ} \mathrm{C}$. Methanol $(\mathrm{MeOH})$ and acetonitrile (ACN) were used as spray solvents. The sprayer potential was varied in the range $1-3 \mathrm{kV}$. Melamine, tetracycline and lincomycin were deposited as solid samples onto each slide by solvent casting ( $1 \mu \mathrm{L}$ of a $100 \mathrm{pmol} / \mu \mathrm{L}$ solution of each of the three components in methanol).

\section{Results}

TM-AFM investigations were carried out to observe surface morphology. Initial TM-AFM images were acquired for plasma-cleaned glass slides, in order to select the smoothest 
side of the slide. Rms roughness $\left(\mathrm{R}_{\mathrm{q}}\right)$ was found to be $0.9 \mathrm{~nm}$, which is within the range reported in the literature, $(0.3-3 \mathrm{~nm})$ [34]. $R_{q}$ was also calculated for the silanized glasses, but no significant increase in surface roughness was observed (Figure 3).

Contact angles are reported in Table 1, as well as the surface energy. As expected, the highest surface free energy was found for g-FOTES and it decreased in the series g-OTES, g-MTES, glass.

XPS spectra of clean glass, g-MTES, g-OTES, and gFOTES were acquired (Supplementary Information). Further peaks found in the survey scans on the functionalized glasses could be attributed to the presence of adsorbed silanes, namely S $2 \mathrm{~s}$ and S 2 p for g-MTES, and F $1 \mathrm{~s}$ for g-FOTES. Starting from g-MTES, three major peaks are identified in the $\mathrm{C} 1 \mathrm{~s}$ spectrum (Figure 4a). The peak centered at $285.0 \mathrm{eV}$ was ascribed to $\mathrm{C}-\mathrm{C}$ and $\mathrm{C}-\mathrm{Si}$ bonds [35-37], whereas carbon atoms bonded to sulfur and oxygen appear at $286.3 \mathrm{eV}$ [38, 39]. A third minor peak, found at about $288 \mathrm{eV}$, may be due to an oxygen-containing compound adsorbed on the sample surface. A further confirmation of the effectiveness of the functionalization was from Si $2 \mathrm{p}$, where the high-resolution spectrum signal was found to contain contributions at 103.0 and $103.7 \mathrm{eV}$ (Figure 4d) ascribable to Si of g-MTES and Si-O of glass [40], respectively. The high-resolution spectrum of $\mathrm{S}$ $2 p$ was fitted with two doublets whose maxima in their $2 \mathrm{p}_{3 / 2}$ components were at 164 and about $168 \mathrm{eV}$, assigned to the presence of well-ordered MTES layers having thiol groups at the top of the surface [41], and to a minor contribution from oxidized sulfur [35, 42, 43] (Supporting Information). For gOTES, similarly to what was observed for g-MTES, C $1 \mathrm{~s}$ showed three major contributions at 285, 286.5, and $288 \mathrm{eV}$ (Figure $4 \mathrm{~b}$ ) and two components for the $\mathrm{Si} 2 \mathrm{p}$ signal at 103.0 and $103.8 \mathrm{eV}$, ascribed to $\mathrm{Si}-\mathrm{C}$ and glass (Figure 4e). In the case of g-FOTES, the $\mathrm{C} 1 \mathrm{~s}$ signal was a convolution of five components at $285.2 \mathrm{eV}$, originating from $\mathrm{C}-\mathrm{C}$ and $\mathrm{C}-\mathrm{Si}$ [4446], and at 286.5, 291.5, 294.0, and $288.0 \mathrm{eV}$ attributable to $\mathrm{C}-\mathrm{O}$ [39], $-\mathrm{CF}_{2}$ and $-\mathrm{CF}_{3}$ groups $[39,44,45]$, as well as a
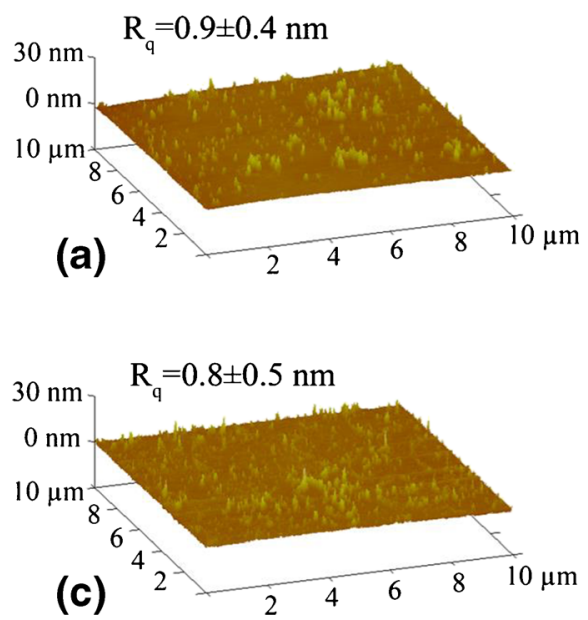

contamination component, respectively (Figure 4c). Two components for the Si $2 \mathrm{p}$ signal at 102.9 and $103.7 \mathrm{eV}$ were ascribed to $\mathrm{Si}-\mathrm{C}[47,48]$ and glass [49], as shown in Figure 4f.

ARXPS investigations were carried out to estimate film thickness, by monitoring the attenuation of Si $2 p$ signal at $103 \mathrm{eV}$ as a function of the emission angle (EA). From the results of $\mathrm{C} / \mathrm{Si}$ versus $\mathrm{EA}$, the thickness of the carbon-chain layer (d) for each functionalized glass is given by the equation I $=\mathrm{I}_{0} \exp (-\mathrm{d} /(\lambda \cos \theta))$, where $\mathrm{I}_{0}$ is the intensity of the emission from the bulk, $\theta$ is the emission angle (with respect to sample normal), $\lambda$ is the inelastic mean free path (referred to the overlayer), and $d$ is the film thickness. From these calculations, it was possible to compare the experimental thickness with the expected one, assuming that a monolayer of silanes had been bound on the surface, with alkyl chains standing perpendicular to the adsorption plane (Table 2).

The three functionalized glasses, as well as clean glass, were used as supports to address surface effects on massspectrometric response when molecules with different acidbase behavior were used as analytes. Melamine was chosen as organic base $\left(\mathrm{pK}_{\mathrm{a}}=8.95\right)$, tetracycline as acid $\left(\mathrm{pK}_{\mathrm{a}}=3.3\right)$, and lincomycin with intermediate $\mathrm{pK}_{\mathrm{a}}$ (7.6). MS experiments were carried out by setting different potential on the sprayer, in order to work on the one hand under electrospray and on the other hand under pneumatically assisted ionization conditions. It was possible to calculate the potential for the onset of the electrospray flow by the approximated formula proposed by Smith [50].

$V_{\text {on }} \approx\left(\frac{r_{c} \gamma \cos \theta}{2 \varepsilon_{o}}\right)^{1 / 2} \ln \left(\frac{4 d}{r_{c}}\right)$

By substituting the values $\varepsilon_{\mathrm{o}}=8.8 \times 10^{-12} \mathrm{~J}^{-1} \mathrm{C}^{2}, \theta=49.3^{\circ}$, [51] $r_{c}=50 \mu \mathrm{m}, d=3 \mu m$ and taking into account methanol (surface tension, $0.0226 \mathrm{~N} \mathrm{~m}^{-1}$ ) and acetonitrile (surface tension, $0.0293 \mathrm{~N} \mathrm{~m}^{-1}$ ) as spray solvents, the equation leads to an onset potential equal to 1.2 and $1.3 \mathrm{kV}$, respectively. Below these potentials, the formation of $\mathrm{MH}^{+}$ions is mainly due to
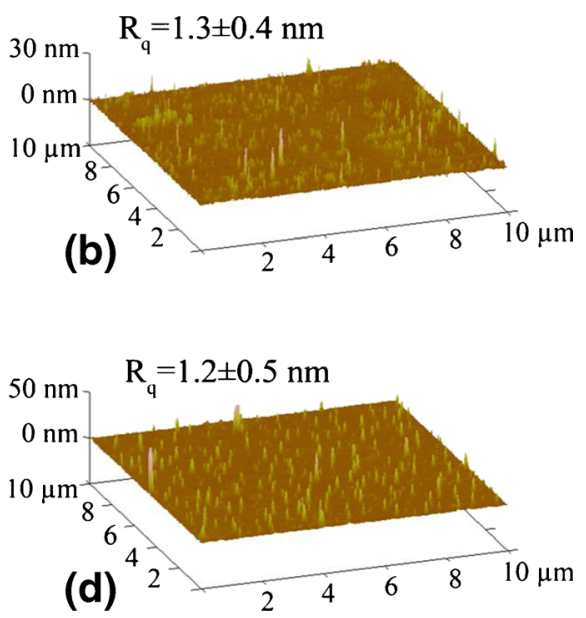

Figure 3. Three-dimensional TM-AFM images of a clean non-coated glass slide (a), g-MTES (b), g-OTES (c), and g-FOTES (d). Results are expressed as mean values $(n=3)$ with standard deviations 
Table 1. Contact Angles and Surface Free Energy Values of Cleaned Glass and Functionalized Slides with the Three Different Silanes

\begin{tabular}{lccc}
\hline \multirow{2}{*}{ Surface } & \multicolumn{2}{c}{ Contact angle $\left(^{\circ}\right)$} & Surface free energy $\left(\mathrm{mJ} \mathrm{m}^{-2}\right)$ \\
\cline { 2 - 3 } & Water & Diiodomethane & \\
\hline Glass & $12 \pm 1$ & $42 \pm 1$ & $71.1 \pm 0.6$ \\
g-MTES & $72 \pm 1$ & $46 \pm 1$ & $39.7 \pm 0.4$ \\
g-OTES & $91 \pm 1$ & $64 \pm 1$ & $27.3 \pm 0.4$ \\
g-FOTES & $106 \pm 2$ & $88 \pm 2$ & $14.5 \pm 0.5$ \\
\hline
\end{tabular}

pneumatic effects, whereas at higher potentials $\mathrm{MH}^{+}$ions are created as a consequence of the formation of a Taylor cone. Therefore, 1 and $3 \mathrm{kV}$ were chosen as sprayer potentials. Desorption/ionization experiments were carried out by spraying solvent onto each self-assembled monolayers (SAM)-coated glass slide and extracted ion currents for each analyte were compared with a three-way ANOVA with interactions $(\alpha=0.05)$, considering surface free energy, spray solvent, and sprayer potential as factors influencing ion current of the analytes. In all cases, $\log _{10}$ data transformation was applied to ensure variance homogeneity among samples as a consequence of a high variability of DESI currents. For all analytes, a significant decrease in ion current versus increasing surface free energy was observed (Figure 5). All factor interactions were found to be significant.

\section{Discussion}

The presence of imperfections on the nano-scale was observed and ascribed to inhomogeneity of the starting glass surfaces and possibly aggregates from in-solution reactions among the silanes. To avoid self-polymerization of silanes, since reactivity with water is well known, in particular when using fluorinated compounds, dry toluene was used as a solvent [27]. Toluene was also used for the surface functionalization for its capability of extracting significant amounts of water from the substrate surface, yielding dense alkylsiloxanes films [52]. Finally it is to be noted that the use of smooth surfaces, expressed in terms of comparable $\mathrm{R}_{\mathrm{q}}$ values, for subsequent DESI-MS experiments, allowed conclusions to be drawn as to how surface chemistry affects ion-formation yield, without any contribution from

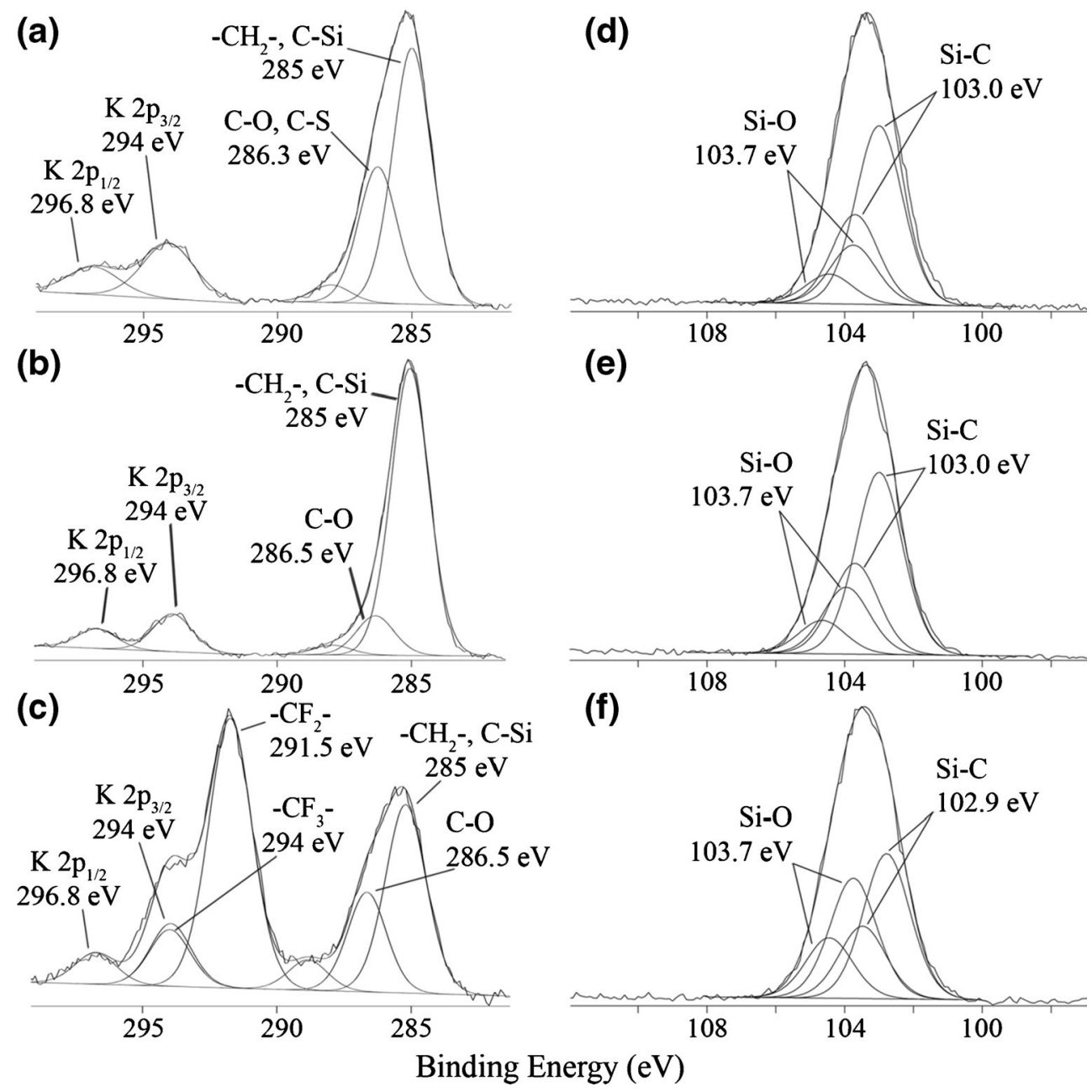

Figure 4. Curve-fitting of high resolution $C$ 1s spectra of samples g-MTES (a), g-OTES (b), and g-FOTES (c); Si 2p spectra of gMTES (d), g-OTES (e), and g-FOTES (f) 
Table 2. Thickness Values for the Three Functionalized Glasses Calculated by Si 2 p Signal Attenuation $(103.0 \pm 0.1 \mathrm{eV})$ as a Function of the Photoemission Angle and Relative Comparison with Expected Values Upon the Hypothesis of the Formation of a Monolayer on Glass Surfaces

\begin{tabular}{lcc}
\hline & \multicolumn{2}{l}{ Thickness (nm) } \\
\cline { 2 - 3 } g-MTES & ARXPS & Expected \\
\cline { 2 - 3 } g-OTES & $1.0 \pm 0.1$ & 0.65 \\
g-FOTES & $1.1 \pm 0.1$ & 1.11 \\
& $1.2 \pm 0.1$ & 1.13 \\
\hline
\end{tabular}

morphologic changes, which will be further discussed in the context of mass spectrometric data.

The maximum density of silane coverage could be readily achieved with each silane, according to previously published results [36, 37, 44, 45, 53, 54]. Further confirmations concerning films structures and homogeneity of the coverage were obtained by XPS.

According to the data reported in Table 2, it might be reasonable to assume that a monolayer of silanes was reached by depositing OTES and FOTES from dry toluene solutions, whereas for MTES the formation of a multilayered system may be assumed. Further insights into surface chemistry were gained by calculating relative ratios among the elements characteristic of each coating. For g-MTES the ratio of $\mathrm{C} 1 \mathrm{~s}$ at $285 \mathrm{eV}$ to $\mathrm{C} 1 \mathrm{~s}$ at $286.3 \mathrm{eV}$ is 1.8 , consistent with MTES condensing on the glass surface, according to previously published results concerning solution-deposited mercapto-silanes [36]. The $\mathrm{C} 1 \mathrm{~s} / \mathrm{O} 1 \mathrm{~s}(533.8 \mathrm{eV})$ ratio was found to be 5.9, which is consistent with previous hypotheses. In addition, the ratio of $\mathrm{C} 1 \mathrm{~s} / \mathrm{S} 2 \mathrm{p}$ was found to be 5.0, consistent with the hypothesis of the multilayered adsorption of silanes, involving ethanesulfonic acid or ethylmercaptan elimination as the multilayer mercaptan grows [35]. These findings support ARXPS observations, which showed a thickness double that expected from a monolayer of MTES molecules. In case of g-OTES, the ratio of $\mathrm{C} 1 \mathrm{~s} / \mathrm{O} 1 \mathrm{~s}(533.8 \mathrm{eV})$ was found to be 9 . For g-FOTES the effectiveness of the coating was confirmed by elemental analysis. The atomic ratio of carbon at $294.0 \mathrm{eV}$ to that at $291.5 \mathrm{eV}$ was constrained so that the ratio $\left(\mathrm{CF}_{3}\right) /\left(\mathrm{CF}_{2}\right)_{5}$ was

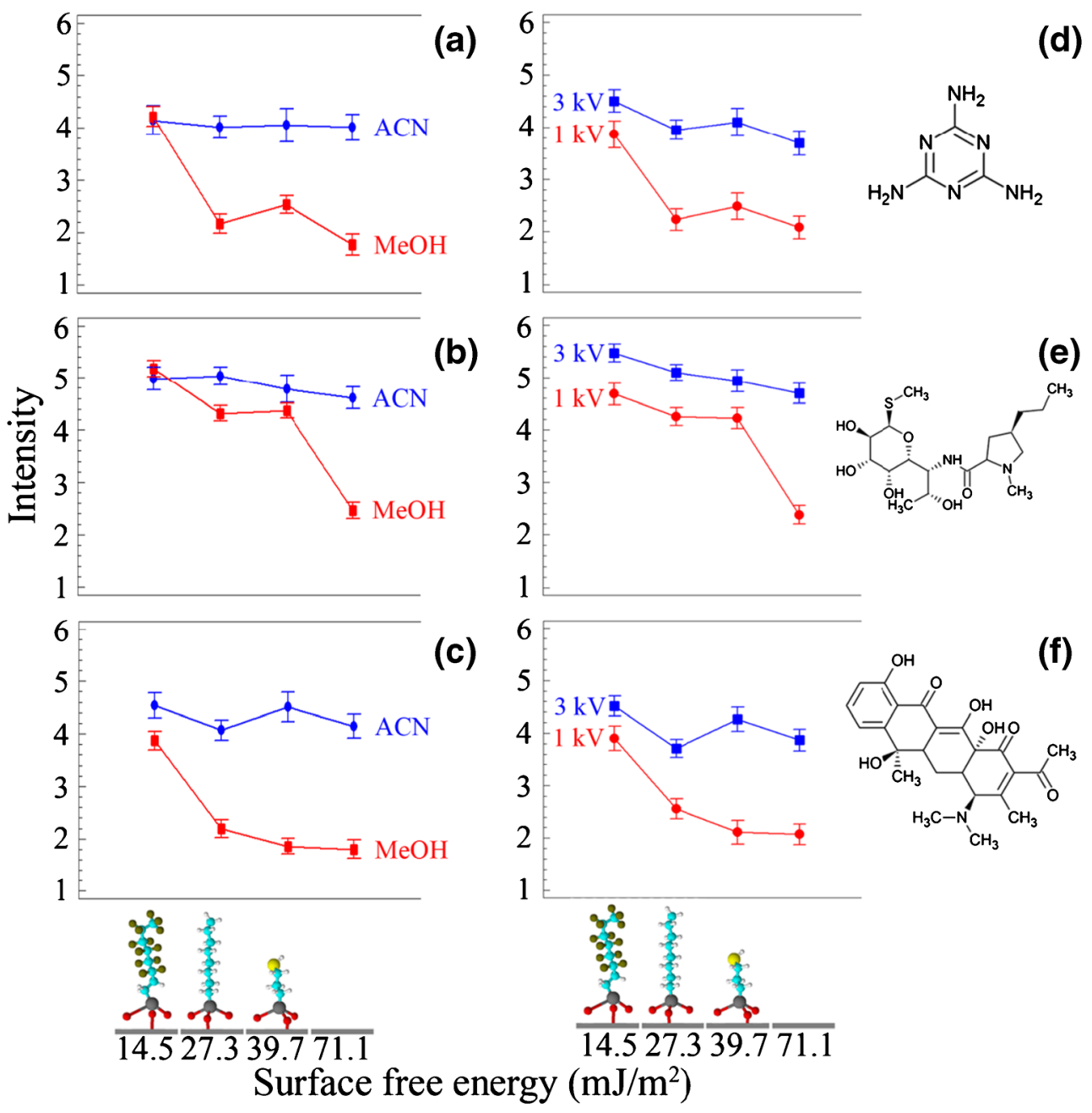

Figure 5. Two-way ANOVA interaction plots of extracted ion current signals (an ion trap was used as detector) of melamine (a), (d), lincomycin (b), (e), tetracycline (c), (f) as a function of surface free energy. At the bottom, associated to surface free energies, models of g-FOTES (left), g-OTES, g-MTES, and glass (right) are reported 
equal to 0.2 and the fit resulted to agree with this assumption. Furthermore for the $\mathrm{C} 1 \mathrm{~s}$ peak originating from $\mathrm{CH}_{2}$ carbons found at $285.2 \mathrm{eV}$ in this spectrum, the observed peak area intensity ratio of $\mathrm{CH}_{2}$ carbons to $\mathrm{CF}_{3}$ carbons is 3.35 , which is larger than the carbon content ratio of $\left(\mathrm{CH}_{2}\right)_{2} /\left(\mathrm{CF}_{3}\right)=2$ in the FOTES molecules, as a consequence of some contamination and of a possible X-ray damage, according to data previously reported in the literature [55]. The $\mathrm{C} 1 \mathrm{~s} / \mathrm{O} 1 \mathrm{~s}(533.8 \mathrm{eV})$ ratio was found to be 6.3, consistent with a partial loss of OEt groups during condensation on the glass.

The idea of taking into account surface free energy to gain insights into DESI process was originated by Volný et al. [8] where this physical quantity is reported as a factor that can influence DESI current, calculated for different supports, ranging from glass to polymethylmethacrylate (PMMA) and polytetrafluoroethylene (PTFE). In that work, capacitor time constants of the equivalent RC circuits for different DESI surfaces were calculated, and the highest value appeared to yield the best analytical response. However, in those experiments, both the electrical and the surface-chemical properties of each material contributed to the analytical response, and electrical resistivity for the three different materials are reported to be significantly different [i.e., $10^{11}$ to $10^{14} \Omega \mathrm{m}$ for glass [56], about $\cdot 10^{9} \Omega \mathrm{m}$ for PMMA [57], and estimated to be above $10^{20} \Omega \mathrm{m}$ for PTFE (DuPont)]. Therefore, to successfully address surface-free-energy effects on DESI efficiency, a functionalization approach by a self-assembly method allowed us to obtain surfaces within a wide range of polarity and, at the same time, to have a constant electrical contribution from the support.

In addition, by having comparable roughness, found to be in the same order of magnitude as shown by TM-AFM investigations, it was possible to avoid contributions to the ionization induced by changes in surface morphology and to address DESI current variations only attributable to surface-chemical modifications.

By moving from a hydrophobic to a hydrophilic surface, under ionization in both desorption electrospray and pneumatically assisted conditions, a significant decrease in ion current was observed, as indicated by the ANOVA results. This is the same result obtained in previous studies [8], except that the results in this case were obtained by desorbing analytes from a support with constant electrical resistivity (i.e., the same bulk electrical properties). As a consequence, it is reasonable to assume that the significant differences in ion current are solely due to wettability effects (i.e., the confinement of the solution layer on a hydrophobic surface leads to a more efficient microextraction step at the solid interface).

Particular attention was devoted to the interaction surface free energy/spray solvent (Figure 5a-c) and surface free energy sprayer potential (Figure $5 \mathrm{~d}-\mathrm{f}$ ) for all analytes (the intensity showed in Figure 5 is the total intensity recorded for a whole acquisition). The effect of the wettability was found to be different when ions were created by ES or only by pneumatically assisted conditions, i.e. above or below the onset of the electrospray $\left(\mathrm{MH}^{+}\right.$formation without or in the presence of a
Taylor cone). These results might be interpreted as follows: as a consequence of a Taylor cone being formed at the capillary tip, by working at a high positive potential (in this case $3 \mathrm{kV}$ ), an accumulation of positive charges takes place, leading to a more efficient ionization process with a consequent apparent leveling effect of the response as a function of surface free energy. Therefore, the use of more hydrophobic surfaces yielded higher signal intensities, regardless of whether the sprayer voltage was below or above the onset of the electrospray. At $5 \mathrm{kV}$, a sprayer potential routinely used in DESI measurements, the signal intensity would behave as at $3 \mathrm{kV}$, as they both are above the onset of the electrospray.

The DESI current was also affected when different solvents were used in the spray, although to a different extent. The highest signal intensity was generated when the potential on the sprayer is $3 \mathrm{kV}$ and the analytes were desorbed and ionized from the most hydrophobic surface. This happened when using both $\mathrm{MeOH}$ and ACN. Small differences were observed between $\mathrm{ACN}$ and $\mathrm{MeOH}$ only when the most hydrophobic surface, g-FOTES, was used. This behavior may be a consequence of different solvent surface tensions (i.e., $22.50 \mathrm{mN} / \mathrm{m}$ for $\mathrm{MeOH}$ and $29.29 \mathrm{mN} / \mathrm{m}$ for $\mathrm{ACN}$ ). In fact, a higher wettability might explain an ion current decrease, induced by a less efficient microextraction at the interface, even though no linear trend was observed. Finally, it might be assumed that surface-activated chemical ionization, induced by SH residues from g-MTES, appears to give a minor contribution to the ionization.

\section{Conclusions}

The results demonstrate that tailoring surfaces allows the performance of a DESI-MS experiment to be enhanced. Furthermore, the role of surface free energy when a DESI-MS experiment is carried out was illustrated. Surface wettability was successfully tuned by self-assembling different silanes onto glass surfaces, as demonstrated by contact angle goniometry, AFM, as well as XPS investigations. It was demonstrated how the analytical response dropped upon using hydrophilic rather than hydrophobic surfaces, both in ESI and in pneumatic ionization conditions, as a consequence of differences in efficiency of the microextraction step at the solid interface.

\section{Acknowledgment}

The authors thank Dr. Maura Crobu for helpful discussions.

\section{References}

1. Venter, A., Nefliu, M., Cooks, R.G.: Ambient desorption ionization mass spectrometry. TrAC Trends Anal. Chem. 27, 284-290 (2008)

2. Takáts, Z., Wiseman, J.M., Cooks, R.G.: Ambient mass spectrometry using desorption electrospray ionization (DESI): instrumentation, mechanisms and applications in forensics, chemistry, and biology. J. Mass Spectrom. 40, 1261-71265 (2005)

3. Gurdak, E., Green, F.M., Rakowska, P.D., Seah, M.P., Salter, T.L., Gilmore, I.S.: VAMAS interlaboratory study for desorption electrospray 
ionization mass spectrometry (DESI MS) intensity repeatability and constancy. Anal. Chem. 86, 9603-9611 (2014)

4. Costa, A.B., Cooks, R.G.: Simulated splashes: elucidating the mechanism of desorption electrospray ionization mass spectrometry. Chem. Phys. Lett. 464, 1-8 (2008)

5. Nefliu, M., Smith, J.N., Venter, A., Cooks, R.G.: Internal energy distributions in desorption electrospray ionization (DESI). J. Am. Soc. Mass Spectrom. 19, 420-427 (2008)

6. Frankevich, V., Nieckarz, R.J., Sagulenko, P.N., Barylyuk, K., Zenobi, R., Levitsky, L.I., Agapov, A.Y., Perlova, T.Y., Gorshkov, M.V., Tarasova, I.A.: Probing the mechanisms of ambient ionization by laser-induced fluorescence spectroscopy. Rapid Commun. Mass Spectrom. 26, 1567$1572(2012)$

7. Venter, A., Sojka, P.E., Cooks, R.G.: Droplet dynamics and ionization mechanisms in desorption electrospray ionization mass spectrometry. Anal. Chem. 78, 8549-8555 (2006)

8. Volný, M., Venter, A., Smith, S.A., Pazzi, M., Cooks, R.G.: Surface effects and electrochemical cell capacitance in desorption electrospray ionization. Analyst 133, 525-531 (2008)

9. Gao, L., Li, G., Cyriac, J., Nie, Z.X., Cooks, R.G.: Imaging of surface charge and the mechanism of desorption electrospray ionization mass spectrometry. J. Phys. Chem. C 114, 5331-5337 (2010)

10. Kaftan, F., Kofroňová, O., Benada, O., Lemr, K., Havlicek, V., Cvacka, J., Volny, M.: Scanning electron microscopic imaging of surface effects in desorption and nano-desorption electrospray ionization. J. Mass Spectrom. 46, 256-261 (2011)

11. Wood, M.C., Busby, D.K., Farnsworth, P.B.: Microscopic imaging of glass surfaces under the effects of desorption electrospray ionization. Anal. Chem. 81, 6407-6415 (2009)

12. Zivolic, F., Zancanaro, F., Favretto, D., Ferrara, S.D., Seraglia, R., Traldi, P.: Pneumatically assisted desorption/ionization: 1 . Some thoughts on the possible ionization mechanism(s). J. Mass Spectrom. 45, 411-420 (2010)

13. Seraglia, R., Molin, L., Isak, I., Traldi, P.: Investigation on the role of pneumatic aspects in electrospray desorption electrospray surface ionization and surface activated chemical ionization. Eur. J. Mass Spectrom. (Chichester, Eng.) 18, 195-203 (2012)

14. Penna, A., Elviri, L., Careri, M., Mangia, A., Predieri, G.: Investigation of novel sol-gel hydrophobic surfaces for desorption electrospray ionizationmass spectrometry analysis. Anal. Bioanal. Chem. 400, 1515-1523 (2011)

15. Ulman, A.: Formation and structure of self-assembled monolayers. Chem. Rev. 96, 1533-1554 (1996)

16. Parikh, A.N., Liedberg, B., Atre, S.V., Ho, M., Allara, D.L.: Correlation of molecular organization and substrate wettability in the self-assembly of nalkylsiloxane monolayers. J. Phys. Chem. 99, 9996-10008 (1995)

17. Brandriss, S., Margel, S.: Synthesis and characterization of self-assembled hydrophobic monolayer coatings on silica colloids. Langmuir 9, 12321240 (1993)

18. Wen, K., Maoz, R., Cohen, H., Sagiv, J., Gibaud, A. Desert, A., Ocko, B.M.: Postassembly chemical modification of a highly ordered organosilane multilayer: new insights into the structure, bonding, and dynamics of self-assembling silane monolayers. ACS Nano 2, 579-599 (2008)

19. Allara, D.L., Parikh, A.N., Rondelez, F.: Evidence for a unique chain organization in long chain silane monolayers deposited on two widely different solid substrates. Langmuir 11, 2357-2360 (1995)

20. Parikh, A.N., Allara, D.L., Ben Azouz, I., Rondelez, F.: An intrinsic relationship between molecular structure in self-assembled n-alkylsiloxane monolayers and deposition temperature. J. Phys. Chem. 98, 7577-7590 (1994)

21. Le Grange, J.D., Markham, J.L., Kurkjian, C.R.: Effects of surface hydration on the deposition of silane monolayers on silica. Langmuir 9, 17491753 (1993)

22. Doudevski, I., Schwartz, D.K.: Concentration dependence of selfassembled monolayer island nucleation and growth. J. Am. Chem. Soc. 123, 6867-6872 (2011)

23. Rozlosnik, N., Gerstenberg, M.C., Larsen, N.B.: Effect of solvents and concentration on the formation of a self-assembled monolayer of octadecylsiloxane on silicon (001). Langmuir 19, 1182-1188 (2003)

24. Krasnoslobodtsev, A.V., Smirnov, S.N.: Effect of water on silanization of silica by trimethoxysilanes. Langmuir 18, 3181-3184 (2002)

25. Leitner, T., Friedbacher, G., Vallant, T., Brunner, H., Mayer, U., Hoffmann, H.: Investigations of the growth of self-assembled octadecylsiloxane monolayers with atomic force microscopy. Mikrochim. Acta 133, 331-336 (2000)
26. Bunker, B.C., Carpick, R.W., Assink, R.A., Thomas, M.L., Hankins, M.G., Voigt, J.A., Sipola, D.,De Boer, M.P., Gulley, G.L.: The Impact of solution agglomeration on the deposition of self-assembled monolayers. Langmuir 16, 7742-7751 (2000)

27. Brzoska, J.B., Ben Azouz, I., Rondelez, F.: Silanization of solid substrates: a step toward reproducibility. Langmuir 10, 4367-4373 (1994)

28. Owens, D.K., Wendt, R.C.: Estimation of the surface free energy of polymers. J. Appl. Polym. Sci. 13, 1741-1747 (1969)

29. Wu, S.: Polymer interface and adhesion. Marcel Dekker, Inc., NY, USA (1982)

30. Powell, C.J.: The physical basis for quantitative surface analysis by auger electron spectroscopy. ASTM International, West Conshohocken, PA, USA (1978)

31. Scofield, J.H.: Hartree-Slater subshell photoionization cross-sections at 1254 and 1487 eV. J. Electron. Spectrosc. Relat. Phenom. 8, 129-137 (1976)

32. Reilman, R.F., Msezane, A., Manson, S.T.: Relative intensities in photoelectron spectroscopy of atoms and molecules. J. Electron. Spectrosc. Relat. Phenom. 8, 389-394 (1976)

33. Seah, M.P., Dench, W.A.: Quantitative electron spectroscopy of surfaces: a standard database for electron inelastic mean free paths in solids. Surf. Interface Anal. 1, 2-11 (1979)

34. Reed, J., Singer, E., Kresbach, G., Schwartz, D.C.: A quantitative study of optical mapping surfaces by atomic force microscopy and restriction endonuclease digestion assays. Anal. Biochem. 259, 80-88 (1998)

35. Senkevich, J.J., Mitchell, C.J., Yang, G.-R., Lu, T.-M.: Surface chemistry of mercaptan and growth of pyridine short-chain alkoxy silane molecular layers. Langmuir 18, 1587-1594 (2002)

36. Singh, J., Whitten, J.E.: Adsorption of 3-mercaptopropyltrimethoxysilane on silicon oxide surfaces and adsorbate interaction with thermally deposited gold. J. Phys. Chem. C 112, 19088-19096 (2008)

37. Chen, J.-J., Struk, K.N., Brennan, A.B...: Surface modification of silicate glass using 3-(mercaptopropyl)trimethoxysilane for thiol-ene polymerization. Langmuir 27, 13754-13761 (2011)

38. Wagner, C.D., Moulder, J.F., Davis, L.E., Riggs, W.M.: Handbook of Xray Photoelectron Spectroscopy. Physical Electronics Division, PerkinElmer Corp., Waltham, MA, USA (1978)

39. Beurer, E., Venkataraman, N.V., Rossi, A., Bachmann, F., Engeli, R., Spencer, N.D.: Orthogonal, three-component, alkanethiol-based surfacechemical gradients on gold. Langmuir 26, 8392-8399 (2010)

40. Wagner, C.D.: Auger and photoelectron line energy relationships in aluminum-oxygen and silicon-oxygen compounds. J. Vac. Sci. Technol. 21, 933 (1982)

41. Hu, M., Noda, S., Tsuji, Y., Okubo, T., Yamaguchi, Y., Komiyama, H.: Effect of interfacial interactions on the initial growth of $\mathrm{Cu}$ on clean $\mathrm{SiO}$ [sub 2] and 3mercaptopropyltrimethoxysilane-modified $\mathrm{SiO}\left[\mathrm{sub}^{2}\right.$ 2] substrates. J. Vac. Sci. Technol. A Vac. Surf. Film 20, 589 (2002)

42. Wang, X., Hu, W., Ramasubramaniam, R., Bernstein, G.H., Snider, G., Lieberman, M.: Formation, characterization, and sub-50-nm patterning of organosilane monolayers with embedded disulfide bonds: an engineered self-assembled monolayer resist for electron-beam lithography. Langmuir 19, 9748-9758 (2003)

43. Zhang, Y.F., Liao, L.S., Chan, W.H., Lee, S.T., Sammynaiken, R., Sham, T.K.: Electronic structure of silicon nanowires: a photoemission and X-ray absorption study. Phys. Rev. B 61, 8298-8305 (2000)

44. Hozumi, A., Ushiyama, K., Sugimura, H., Takai, O.: Fluoroalkylsilane monolayers formed by chemical vapor surface modification on hydroxylated oxide surfaces. Langmuir 15, 7600-7604 (1999)

45. Yokota, K., Takai, K., Enoki, T.: Carrier control of graphene driven by the proximity effect of functionalized self-assembled monolayers. Nano Lett. 11, 3669-3675 (2011)

46. Beamson, G., Alexander, M.R.: Angle-resolved XPS of fluorinated and semi-fluorinated side-chain polymers. Surf. Interface Anal. 36, 323-333 (2004)

47. Alexander, M.R., Short, R.D., Jones, F.R., Michaeli, W., Blomfield, C.J.: A study of HMDSO/O2 plasma deposits using a high-sensitivity and -energy resolution XPS instrument: curve fitting of the Si 2p core level. Appl. Surf. Sci. 137, 179-183 (1999)

48. Azioune, A., Marcozzi, M., Revello, V., Pireaux, J.-J.: Deposition of polysiloxane-like nanofilms onto an aluminium alloy by plasma polymerized hexamethyldisiloxane: characterization by XPS and contact angle measurements. Surf. Interface Anal. 39, 615-623 (2007)

49. Beamson, G., Briggs, D.: High resolution XPS of organic polymers: the Scienta ESCA300 Database. J. Chem. Educ., 70, p A25 (1993) 
50. Smith, D.P.H.: The electrohydrodynamic atomization of liquids. IEEE Trans. Ind. Appl. IA-22, 527-535 (1986)

51. Taylor, G.I., McEvan, A.D.: The stability of a horizontal fluid interface in a vertical electric field. J. Fluid Mech. 22, 1 (1965)

52. McGovern, M.E., Kallury, K.M.R., Thompson, M.: Role of solvent on the silanization of glass with octadecyltrichlorosilane. Langmuir 10, 36073614 (1994)

53. Can, K., Ozmen, M., Gurfidan, L., Gubbuk, I.H., Kaymak, E., Ersoz, M., Ozbek, Z., Capan, R.: Fabrication of octyltriethoxysilane Langmuir-Blodgett thin film. J. Optoelectron. Adv. Mater. 12, $1552-1555$ (2010)
54. Spori, D.M., Drobek, T., Zürcher, S., Spencer, N.D.: Cassie-state wetting investigated by means of a hole-to-pillar density gradient. Langmuir $\mathbf{2 6}$, 9465-9473 (2010)

55. Storp, S.: Radiation damage during surface analysis. Spectrochim. Acta B At. Spectrosc. 40, 745-756 (1985)

56. Serway, R.A.: Principles of physics. Saunders College Pub., Philadelphia, PA, USA (1998)

57. Mudarra, M., Díaz-Calleja, R., Belana, J., Canadas, J.C,, Diego, J.A., Sellares, J., Sanchis, M.J.: Study of space charge relaxation in PMMA at high temperatures by dynamic electrical analysis. Polymer (Guildf) 42, $1647-1651(2001)$ 